\title{
Impaired cerebral hemodynamics in late-onset depression: computed tomography angiography, computed tomography perfusion, and magnetic resonance imaging evaluation
}

\author{
Jinhong Wang ${ }^{1 \#}$, Renren $\mathrm{Li}^{2 \#}$, Meng Liu' ${ }^{2}$ Zhiyu $\mathrm{Nie}^{2}$, Lingjing Jin ${ }^{2}$, Zheng $\mathrm{Lu}^{3}$, Yunxia $\mathrm{Li}^{2}$ \\ ${ }^{1}$ Department of Medical Imaging, Shanghai Mental Health Center, Shanghai Jiao Tong University School of Medicine, Shanghai, China; \\ ${ }^{2}$ Department of Neurology, Tongji Hospital, ${ }^{3}$ Department of Psychiatry, Tongji Hospital, Tongji University School of Medicine, Tongji University, \\ Shanghai, China
}

"These authors contributed equally to this work.

Correspondence to: Yunxia Li. Department of Neurology, Tongji Hospital, Tongji University School of Medicine, Tongji University, 389 Xincun Road, Shanghai 200065, China. Email: doctorliyunxia@163.com.

Background: Late-onset depression (LOD) is often difficult to recognize when there is an absence of a family history of depression and less severe psychopathology. Increasing evidence has shown that the development and course of LOD symptomatology are associated with cerebrovascular comorbidities and cerebral microvascular lesions. This study was designed to evaluate the associations of LOD with macrovascular and microvascular changes in the brain by using a multi-imaging method, including computed tomography angiography (CTA), CT perfusion (CTP), and magnetic resonance imaging (MRI), to explore the course and pathomechanism of LOD.

Methods: A total of 116 participants were divided into two groups. Participants older than 60 years who met the diagnostic criteria of depression [International Classification of Diseases (ICD), 10th Edition] were enrolled in the LOD group, and the remainder were age- and sex-matched into the control group. The cognitive/mood status of all participants was evaluated by an experienced neuropsychologist. Global and regional mean cerebral blood flow (CBF) were measured by CT cerebrovascular perfusion imaging; the stenosis of the bilateral intracranial large arteries (internal carotid artery, anterior cerebral artery, middle cerebral artery, posterior cerebral artery, and vertebral artery) was recorded by CTA; regional white matter hyperintensity (WMH) loads were evaluated by fluid-attenuated inversion recovery (FLAIR) MRI; and the Hamilton Depression Scale (HAMD) was used to evaluate depression status.

Results: Our key findings were the following: (I) participants in the LOD group were more prone to intracranial arterial stenosis $(81.1 \%$ vs. $74.6 \%)$, had more severe stenotic arteries compared with controls $(\mathrm{Z}=2.024, \mathrm{P}<0.05)$, and significantly more participants with LOD had severe stenosis of the middle cerebral artery (MCA) $(9.4 \%$ vs. $0 \%, \mathrm{P}<0.05)$; (II) there was a significant difference in hypoperfusion of the frontal and parietal lobes superposed on global cerebral hypoperfusion between the two groups $(\mathrm{P}<0.001)$; (III) and there was a significant difference in high WMH loads in deep white matter (DWM) between the two groups $(\mathrm{P}<0.05)$. Conclusions: A low global or regional perfusion state, moderate-to-severe stenosis of MCAs, and high WMH loads could be used as imaging biomarkers to indicate diffuse or localized cerebral macrovascular and microvascular pathology in LOD.

Keywords: Late-onset depression (LOD); white matter hyperintensity (WMH); cerebral blood flow (CBF); computed tomography perfusion (CTP); magnetic resonance imaging (MRI); computed tomography angiography (CTA)

Submitted May 04, 2019. Accepted for publication Jun 16, 2020.

doi: 10.21037/qims-19-402

View this article at: http://dx.doi.org/10.21037/qims-19-402 


\section{Introduction}

Depression, similar to diabetes and hypertension, is a serious but treatable medical condition. Older adults are at an increased risk of experiencing depression. Lateonset depression (LOD) is a subtype of depression that often occurs later in life and is characterized by depressive symptoms and cerebrovascular disease as diagnosed by neuroimaging (1-3). LOD is often more difficult to recognize than other subtypes of depression, and it is more difficult for patients to accept their condition and receive treatment, resulting in poor quality of life, social isolation, and physical impairment. These LOD patients usually do not have a family history of depression and exhibit less severe psychopathology and a limited response to treatment, resulting in overall poor outcomes (4).

LOD often occurs in the context of vascular disease and can be defined as depression that occurs after the age of 60 years, although age of onset and definition of cutoff may vary (5). LOD has also been called vascular depression. Although they have been considered different diseases, some researchers have recently posited that the concept of vascular depression should be confined to $\operatorname{LOD}(6,7)$, as both exhibit the same microvascular lesions, cerebrovascular comorbidities, and white matter hyperintensities (WMHs) $(8,9)$. Vascular depression may be diagnosed by a combination of the following: depressive ideation, psychomotor agitation, apathy, executive dysfunction, and abnormalities in the white matter as imaged by magnetic resonance imaging (MRI) (8). Subcortical ischemic lesions and lesion location may play a role in the pathogenesis of LOD (10-13), and the most severe lesions tend to be in the frontal lobe and basal ganglia regions (14). A recent study on MRI-defined vascular depression in community populations reported that deep WMHs are a major risk factor for developing depressive disorders. Moreover, WMHs are an independent predictor of depressive disorders (15).

Multimodality imaging assessment of mood disorders has made a unique contribution to the identification of impaired cerebral hemodynamics. Several studies have found that depression severity is negatively correlated with decreased regional cerebral blood flow (rCBF) (16-18). MRI and transcranial doppler ultrasound (TCD) findings suggest that small vessels, microvascular lesions, and WMHs may affect the neural circuitry of emotional regulation, triggering depressive symptoms. These findings corroborate those obtained by electrophysiological techniques, namely transcranial magnetic stimulation, administered in vascular depression (19-21). Another possibility is that chronic accumulated microvascular burden may induce vascular depression $(22,23)$.

Challenges remain in the study of the pathophysiology of vascular depression and detection of the disease in the general population (24). Few studies have investigated the relationships between vascular depression, hemodynamics (macrovascular), and WMHs (microvascular) to explore the course and pathomechanism of vascular depression. The purpose of this study was to evaluate the associations of LOD with macrovascular and microvascular changes in the brain using a combination of computed tomography angiography (CTA), CT perfusion (CTP), and MRI.

\section{Methods}

\section{Study population}

In all, 116 participants were recruited from the Department of Neurology and Memory Clinic in Shanghai Tongji Hospital between December 2015 and December 2017. These participants were divided into two groups: individuals older than 60 years who met the diagnostic criteria for depression according to the International Classification of Diseases (ICD, 10th Edition) were enrolled in the LOD group (depression group), and the remainder were ageand sex-matched as controls (control group). The LOD group comprised 53 participants with LOD, 20 males and 33 females, with an average age of $72.45 \pm 9.44$ years. The control group comprised 63 participants, 29 males and 34 females, with an average age of $69.08 \pm 9.08$ years. All participants underwent CTA, CTP, and MRI to evaluate macrovessels, hemodynamics, and microvessels. All participants completed informed consent forms. This study was reviewed and approved by the Institutional Review Board at Tongji Hospital.

Participant inclusion criteria included the following: (I) the ability to cooperate throughout all examinations without being hindered by severe hearing or visual impairment; (II) the absence of severe heart, liver, and kidney disease; (III) no history of central nervous system disease; (IV) no history of alcohol or drug dependence or other mental illness; (V) no MRI contraindications; (VI) no allergies to iodine; (VII) no cerebral infarction or hemorrhage confirmed by CT or MRI examination.

\section{Participant information}

The clinical information collected from all participants included the following: sex, age, neurological examination, 
and vascular risk factors. Vascular risk factors included a family history of cerebrovascular disease (primarily cerebral infarction), hypertension, diabetes mellitus, hyperlipidemia, anemia, ischemic heart disease, smoking [at least 1 cigarette per day for more than 1 year as per the 1984 World Health Organization (WHO) standard on smoking survey methods], and alcohol abuse (alcohol consumption more than $50 \mathrm{~g} / \mathrm{d}$, or more than 2 bottles of beer in 1 day more than 5 days a week). In addition, all participants were evaluated by an experienced neuropsychologist and a detailed medical history was taken. If necessary, caregivers were asked to obtain more reliable psychological scores to determine the depressed emotional state of participants with more accuracy, and the relevant information of caregivers was collected in these cases.

\section{The Hamilton Depression Scale (HAMD) evaluation}

All participants underwent neuropsychological assessment. The HAMD was used to evaluate the depression status of the participants. These assessments were all performed in a quiet room by the same qualified neuropsychologist, and all participants were emotionally stable when the assessments were performed.

\section{CT scanning and MRI acquisition $(25,26)$}

All participants underwent whole-brain perfusion imaging with a 320-detector row CT system (Aquilion ONE; Toshiba Medical Systems, Tokyo, Japan) and MRI scanning with a 3.0T MR system (Magnetom Verio, Siemens Medical Systems, Erlangen, Germany).

The CTA parameters were as follows: (I) Toshiba Aquilion ONE 320 detector row CT for whole-brain perfusion imaging: coverage, $140 \mathrm{~mm}$; volume scanning thickness, $0.5 \mathrm{~mm}$; field of vision (FOV), $220 \mathrm{~mm}$; matrix, $512 \times 512$; tube voltage, $100 \mathrm{kV}$; tube current, $150 \mathrm{~mA}$. First, $50 \mathrm{~mL}$ of contrast medium (iohexol $350 \mathrm{mg} \mathrm{I} / \mathrm{mL}$ ) was injected at a flow rate of $5 \mathrm{~mL} / \mathrm{s}$, and the first scan was carried out with a 7-second delay after the injection of contrast medium. The continuous intermittent scan was then carried out with an interval of 2 seconds. The arterial phase reached a peak between 18-28 seconds. During the venous phase, intermittent scanning was performed every 5 seconds. The rotation time was 0.5 seconds, and the total scan duration was 60 seconds. Finally, the whole brain dynamic volume data of 19 phases were obtained.

The fluid-attenuated inversion recovery (FLAIR) sequence MRI was performed with the following parameters: time of repetition (TR), 5,000 ms; time of echo (TE), $94 \mathrm{~ms}$; time of inversion (TI), 1,800 ms; slice thickness, $5 \mathrm{~mm}$; slice interval, $1 \mathrm{~mm}$; field of view (FOV), $230 \mathrm{~mm}$. The AC-PC line served as a baseline, and a total of 23 slices were acquired.

\section{Construction of CBF map and generation of CTA data (25)}

All CTP images of sufficient quality were preprocessed with Perfusion Mismatch Analyzer (PMA, Ver. 5.0.0.0.) software to calculate perfusion maps of CBF. Calculations were conducted using PMA parameters.

To perform a quantitative analysis, all transformed data were imported into Mango Ver. 4.0.1 software to draw regions of interest (ROIs). All volumetric data were inserted into a three-dimensional CTA imaging system (Toshiba) for subtraction angiography automatically to produce threedimensional images.

\section{Assessment of intracranial artery stenosis $(25,27)$}

The warfarin-aspirin symptomatic intracranial disease (WASID) method (28) was used to assess intracranial artery stenosis. The stenosis rate was calculated as follows: stenosis rate $=1-($ diameter of stenotic artery/diameter of distal normal artery) $\times 100 \%$. The bilateral internal carotid artery (ICA), anterior cerebral artery (ACA), middle cerebral artery (MCA), posterior cerebral artery (PCA), and vertebral artery (VA) were assessed for stenosis. Intracranial artery stenosis was evaluated by an experienced neurologist.

\section{Cerebral CTP assessment $(25,26)$}

All data transformed by PMA were imported into Mango software to manually draw ROIs on the lateral ventricle layer and to obtain the CBF values of the frontal, temporal, parietal, and occipital lobes, and global hemisphere area. The steps we took, including the addition of template formulation and ROI selection, are detailed as follows: collect the CTP image data of the participants; evaluate the quality of the perfusion images; eliminate images with heavy motion artifacts and skull base artifacts; use PMA software to calculate the perfusion image of the total CBF; use the Circle of Willis as the reference level with the software automatically selecting 10 artery input functions (AIFs), and the vein output function automatically selecting the intracranial vein above the skull base. The deconvolution 


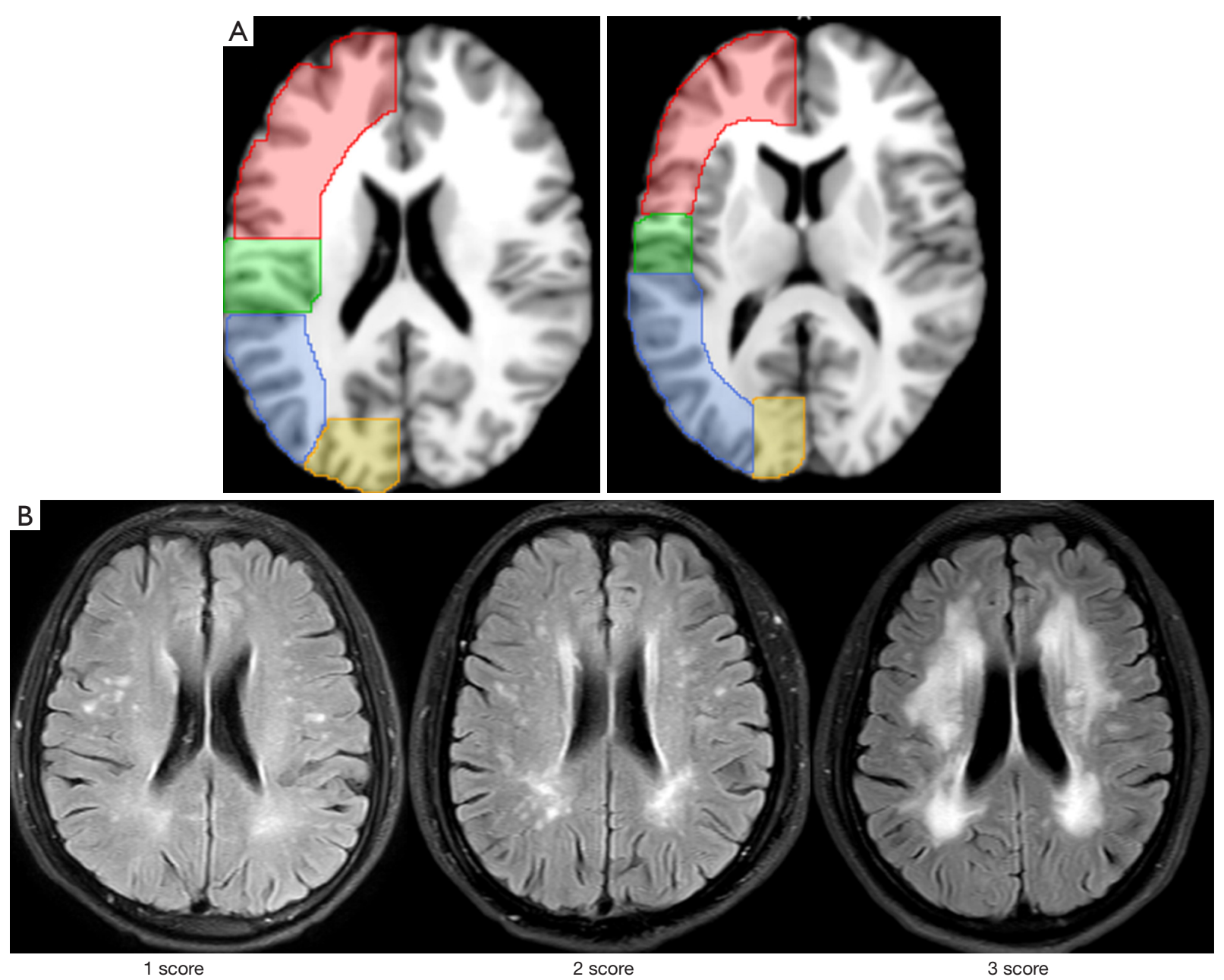

Figure 1 Sketches of ROIs for cerebral perfusion and Kim score of white matter lesions. (A) The ROIs for cerebral perfusion: red, area of frontal lobe; green, area of parietal lobe; blue, area of temporal lobe; yellow, area of occipital lobe; (B) the Kim score in detail: 1 = small cap or thin lining lesion or punctuate lesion; 2 = larger cap or smooth halo lesion or more punctuate lesion with confluence; $3=$ extending cap or irregular extended hyperintense or lesion with massive confluence.

method was then used to calculate the perfusion diagram, with PMA parameters remaining at the default value.

The first step was to select a CTA original data map with a good position on the template. Secondly, two typical images were selected: the lateral ventricle body level (the middle level of the line between the upper and lower edge of the lateral ventricle), and the basal ganglia level (the line between the frontal and posterior horns of lateral ventricle). The third step was to sketch the template ROI on the above two levels. The scope of the ROIs was carefully divided according to relevant information available on this website: https://www.imaios.com/en/e-Anatomy/Head-andNeck/Brain-MRI-in-axial-slices. On this website, under "Anatomical parts", we selected and referred to "Regions:
Cerebral lobes-Brainstem”. Information was also obtained with respect to brain lobes from https://radiopaedia.org/ (ROI are shown in the CTP images at Figure 1A). The fourth step was to adjust the CTA images of each patient to be consistent with the template. Lastly, the original ROI were loaded into the normalized CTP images and the ROIs were fine-tuned to avoid the sinuses and ventricles as much as possible to reduce measurement error. The CBF value of each ROI was obtained at each layer.

\section{WMH assessment (26)}

WMHs were evaluated according the classification system proposed by Kim (29). The Kim score for each WMH 
Table 1 Demographic and clinical characteristics regarding the risk factors of late onset depression (LOD)

\begin{tabular}{lccc}
\hline Variables & Control group $(\mathrm{n}=63)$ & Depression group $(\mathrm{n}=53)$ & $\mathrm{P}$ value \\
\hline Age (years), mean $\pm \mathrm{SD}$ & $69.08 \pm 9.08$ & $72.45 \pm 9.44$ & 0.053 \\
HAMD score, mean $\pm \mathrm{SD}$ & $5.32 \pm 2.40$ & $14.00 \pm 3.92$ & $<0.001$ \\
Female, $\mathrm{n}(\%)$ & $34(54.0)$ & $33(62.3)$ & 0.368 \\
Hypertension, $\mathrm{n}(\%)$ & $32(50.8)$ & $33(62.3)$ & 0.215 \\
Diabetes mellitus, $\mathrm{n}(\%)$ & $8(12.7)$ & $6(11.3)$ & 0.821 \\
Hyperlipidemia, $\mathrm{n}(\%)$ & $33(52.4)$ & $27(50.9)$ & 0.877 \\
Ischemic heart disease, $\mathrm{n}(\%)$ & $5(7.9)$ & $4(7.5)$ & 1.000 \\
Family history of cerebrovascular disease, $\mathrm{n}(\%)$ & $1(1.6)$ & $3(5.7)$ & 0.492 \\
Anemia, $\mathrm{n}$ (\%) & $4(6.3)$ & $7(13.2)$ & 0.209 \\
Smoking, $\mathrm{n}(\%)$ & $5(7.9)$ & $1(1.9)$ & 0.296 \\
Alcohol abuse, $\mathrm{n}(\%)$ & $2(3.2)$ & $0(0.0)$ & 0.554 \\
\hline
\end{tabular}

ranges from 0 to 3 and the specific scoring calculated for juxtaventricular WMH (JVWMH) was the following: $0=$ no $W M H ; 1=$ small cap or thin lining lesion; 2 = larger cap or smooth halo lesion; $3=$ extending cap or irregular extended hyperintense. The following scoring was calculated for periventricular WMH (PVWMH), deep WMH (DWMH), and juxtacortical WMH (JCWMH): $0=$ no $\mathrm{WMH} ; 1=$ punctuate lesion; 2 = increased punctuate lesion with confluence; 3 = lesion with massive confluence (Figure 1B). The final score of each subtype was the sum of the two sides with a range of 0 to 6 . If the Kim score $\geq 3$, we categorized this subtype of WMH as a moderate-to-severe lesion. The images generated from all participants were graded for WMHs by the same experienced investigator who was unaware of the HAMD results.

\section{Statistical analysis}

All statistical analyses were performed with IBM SPSS Statistics 20.0. The differences in CBF between the two groups were compared using independent samples $t$-test, and the differences in Kim score were compared by a nonparametric test. The incidence of WMHs between the two groups were compared by Chi-square test. The association between two variables was evaluated with Spearman's correlation analysis. A two-sided $\mathrm{P}$ value of $<0.05$ was considered statistically significant.

\section{Results}

\section{Clinical characteristics}

To minimize the bias between the control and depression groups, age and sex was matched for all participants. Therefore, no significant demographic variations were found between the control and depression groups. Other confounding factors, such as hypertension, diabetes, hyperlipidemia, coronaropathy, family history of stroke, anemia, smoking, and alcohol abuse were tightly controlled between the control and study groups. As expected, there was a significant difference in HAMD score between the control and depression group $(\mathrm{P}<0.001$, Table 1$)$.

\section{CBF comparisons of $L O D$ with cerebral macro- and microcirculation}

Figure 2 shows a comparison of the percentage of intracranial arterial stenosis in several intracranial large arteries between the control and depression group. These results showed that the depression group was more prone to intracranial arterial stenosis (diameter of a stenotic artery $\leq 50 \%$ diameter of a normal artery) $(81.1 \%$ vs. $74.6 \%$, $\mathrm{P}=0.40)$ and had more severe stenotic arteries than the control group $(\mathrm{Z}=2.024, \mathrm{P}<0.05)$ (Figure $2 A)$. Additionally, the percentage of moderate-to-severe stenotic MCAs in the depression group was significantly higher than that in 
A
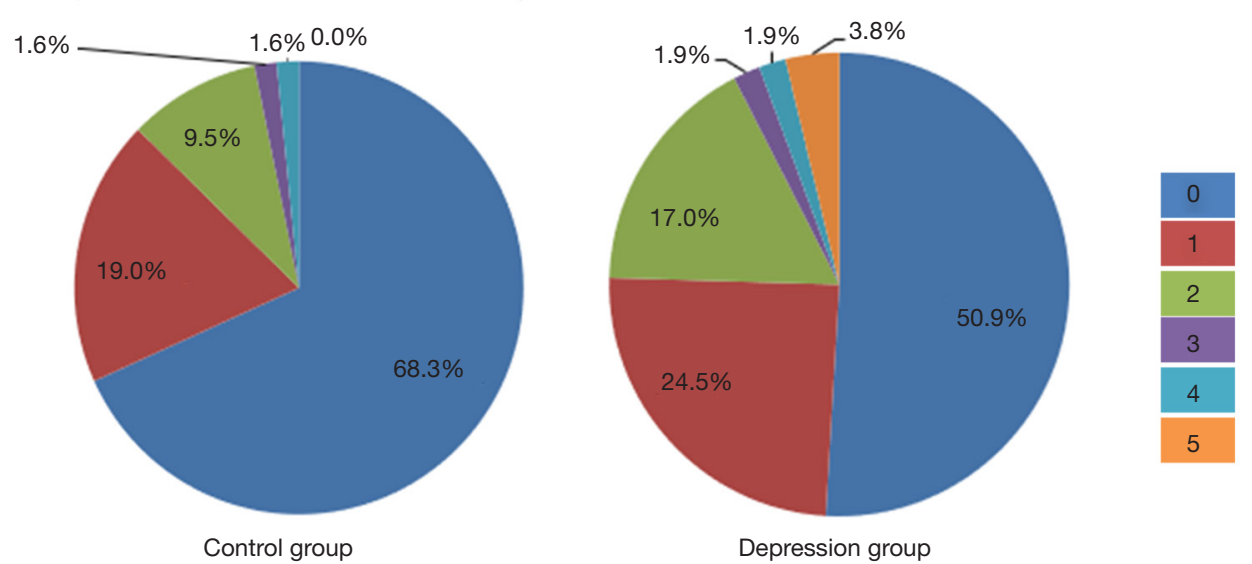

B

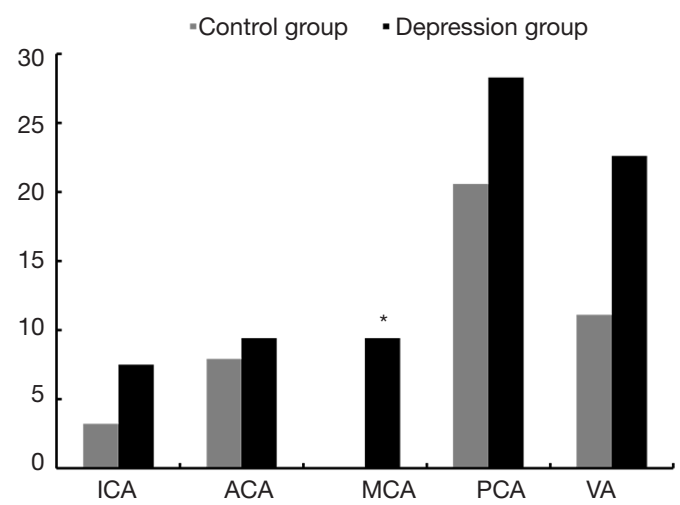

Figure 2 Comparison of moderate-to-severe stenotic arteries between control group and LOD group. (A) The blue area represents the percentage of mild stenotic arteries (diameter of stenotic artery $\leq 50 \%$ diameter of normal artery) (68\% vs. 51\%). The non-blue area represents the percentage of moderate-to-severe stenotic arteries (diameter of stenotic artery $\geq 50 \%$ diameter of normal artery). The numbers 0-5 refer to the number of moderate-to-severe stenotic arteries. (B) The comparison of percentage of moderate-to-severe stenotic arteries, including the internal carotid artery (ICA), anterior cerebral artery (ACA), middle cerebral artery (MCA), posterior cerebral artery (PCA), and vertebral artery (VA) between the control and depression group. *, $\mathrm{P}<0.05$.

control group $(9.4 \%$ vs. $0 \%, \mathrm{P}<0.05)$ (Figure $2 B)$.

CTP imaging reflects brain tissue microcirculation. Thus, using CTP to investigate CBF, we found significantly decreased blood flow in the temporal lobe $(\downarrow 15 \%)$ and occipital lobe $(\downarrow 14.9 \%)$ areas of participants with LOD compared to normal controls $(\mathrm{P}<0.05)$. We further found more significantly decreased blood flow in the frontal lobe ( $\downarrow 16.7 \%)$, parietal lobe $(\downarrow 16.3 \%)$ and global hemisphere ( $\downarrow 16.7 \%)$ of participants with LOD compared to controls $(\mathrm{P}<0.001)$ (Table 2).

Taking into account the two major risk factors of cerebrovascular disease, hypertension and diabetes, we further divided the participants into two groups: a group with hypertension and/or diabetes (67 participants in the mixed group), and a group without hypertension and/or diabetes
(49 participants in the mixed group). The results of the analysis within the mixed groups were consistent with the overall results $(82.9 \%$ vs. $87.5 \%, \mathrm{P}>0.05$ for the percentage of stenotic arteries). For participants with hypertension and/or diabetes, the CBF values of those in the depression group were lower than that of the control group (24.94 vs. $30.35 \mathrm{~mL} / 100 \mathrm{~g} / \mathrm{min}$ in the frontal lobe, $\mathrm{P}<0.05 ; 27.01$ vs. $32.79 \mathrm{~mL} / 100 \mathrm{~g} / \mathrm{min}$ in the parietal lobe, $\mathrm{P}<0.05 ; 45.20$ vs. $54.03 \mathrm{~mL} / 100 \mathrm{~g} / \mathrm{min}$ in the temporal lobe, $\mathrm{P}<0.05 ; 28.31$ vs. $34.76 \mathrm{~mL} / 100 \mathrm{~g} / \mathrm{min}$ in the occipital lobe, $\mathrm{P}<0.05$; and 26.27 vs. $32.05 \mathrm{~mL} / 100 \mathrm{~g} / \mathrm{min}$ in global hemisphere, $\mathrm{P}<0.05$ ). For participants in the group without cerebrovascular disease risk factors, the CBF values of those in the depression group were lower than those of the control group (26.63 vs. $30.87 \mathrm{~mL} / 100 \mathrm{~g} / \mathrm{min}$ in the frontal lobe, $\mathrm{P}<0.05 ; 29.22$ vs. 
Table 2 The distribution of CBF in ROI in control group and late-onset depression (LOD) group

\begin{tabular}{lccc}
\hline CBF $(\mathrm{mL} / 100 \mathrm{~g} / \mathrm{min})$ & Control group $(\mathrm{n}=63)$ & Depression group $(\mathrm{n}=53)$ & $\mathrm{P}$ value \\
\hline Frontal lobe & $30.60 \pm 5.62$ & $25.51 \pm 7.77$ & $46.61 \pm 15.33$ \\
Temporal lobe & $54.77 \pm 11.07$ & $27.76 \pm 8.63$ & $0.002^{*}$ \\
Parietal lobe & $33.19 \pm 5.79$ & $29.06 \pm 9.35$ & $<0.001^{*}$ \\
Occipital lobe & $34.19 \pm 6.28$ & $26.92 \pm 8.16$ & $0.001^{*}$ \\
Global hemisphere & $32.28 \pm 5.94$ & $<0.001^{*}$ \\
\hline
\end{tabular}

Values of $\mathrm{CBF}$ are reported as mean $\pm \mathrm{SD} .{ }^{*}, \mathrm{P}<0.05$.

$33.60 \mathrm{~mL} / 100 \mathrm{~g} / \mathrm{min}$ in the parietal lobe, $\mathrm{P}<0.05 ; 49.37$ vs. $55.54 \mathrm{~mL} / 100 \mathrm{~g} / \mathrm{min}$ in the temporal lobe, $\mathrm{P}>0.05 ; 30.53$ vs. $33.61 \mathrm{~mL} / 100 \mathrm{~g} / \mathrm{min}$ in the occipital lobe, $\mathrm{P}>0.05$; and 28.18 vs. $32.52 \mathrm{~mL} / 100 \mathrm{~g} / \mathrm{min}$ in the global hemisphere, $\mathrm{P}<0.05$ ).

\section{Correlations between CBF, WMH load, and HAMD score}

HAMD scores were negatively correlated with CBF in the global hemisphere $(\mathrm{r}=-0.223, \mathrm{P}<0.05)$, frontal lobe $(\mathrm{r}=-0.226, \mathrm{P}<0.05)$, parietal lobe $(\mathrm{r}=-0.198, \mathrm{P}<0.05)$, and temporal lobe $(\mathrm{r}=-0.187, \mathrm{P}<0.05)$. No significant correlation was found between HAMD score and CBF in the occipital lobe (Figure 3). No significant correlation between HAMD score and regional WMH loads was identified $(\mathrm{P}>0.05)$.

\section{Comparison of regional WMH load}

A significant difference was found between the control and depression groups in the percentage of WMH load in DWM $(\mathrm{P}<0.05)$. Interestingly, no significant difference was observed in the percentage of WMH load in JCWM, PVWM, and JVWM between the two groups $(\mathrm{P}>0.05)$. The percentage of regional WMH load was compared between the control and depression groups, and a higher percentage of moderate-to-severe WMH load in DWM was found in the depression group (Figure 4) compared to the control group $(35.8 \%$ vs. $14.3 \%, \mathrm{P}<0.01)$.

\section{Discussion}

The major findings of our study include the following: (I) significant stenosis in the MCAs of the depression group; (II) highly significant hypoperfusion in the frontal and parietal lobes superposed on global cerebral hypoperfusion in the depression group; (III) and a significant difference in high DWMH loads between the two groups. Our data indicated that moderate-to-severe cerebral damage in the DWM combined with significant hypoperfusion of the frontal and parietal lobes may either contribute to the pathogenesis of LOD or aggravate the disease. These findings demonstrate the relationships between cerebral hypoperfusion, $\mathrm{WMH}$ loads, and LOD. These hemodynamic changes are a result of impaired cerebral macrocirculation and microcirculation, and ischemic damage to cerebral small vessels. This was recently demonstrated in a TCD study of patients with WMHs and a cognitive profile of mild vascular cognitive impairment (30).

LOD has important implications for the understanding of vascular dementia pathogenesis, as well as its treatment and prevention; LOD itself has been considered a major risk factor for vascular dementia (31-33), but the pathophysiology of LOD is not yet well understood. Current theories are based on studies that investigate risk factors such as age, female sex, related cerebrovascular disease, bereavement, sleep disturbance, genetics, social dysfunction, WMHs, and subcortical ischemic brain damage $(3,11,12,34,35)$. Traditional risk factors of LOD or vascular depression were considered in our study during participant selection to minimize bias. Neuroimaging studies have provided mounting evidence to support the correlation between cerebro-cardiovascular disease and LOD. CTA, magnetic resonance angiography (MRA), and perfusion neuroimaging can facilitate study of the pathological and vascular basis of LOD, as these neuroimaging techniques reflect either cerebral macrocirculation or microcirculation conditions.

Vascular dysregulation is common in vascular depression. Reduction of CBF can impair regional or global brain function, contributing to depressive symptoms $(36,37)$. Large vessel cerebral vascular disease affects macrocirculation and is characterized by the presence of stenosis in major cerebral arteries. Our study showed more 

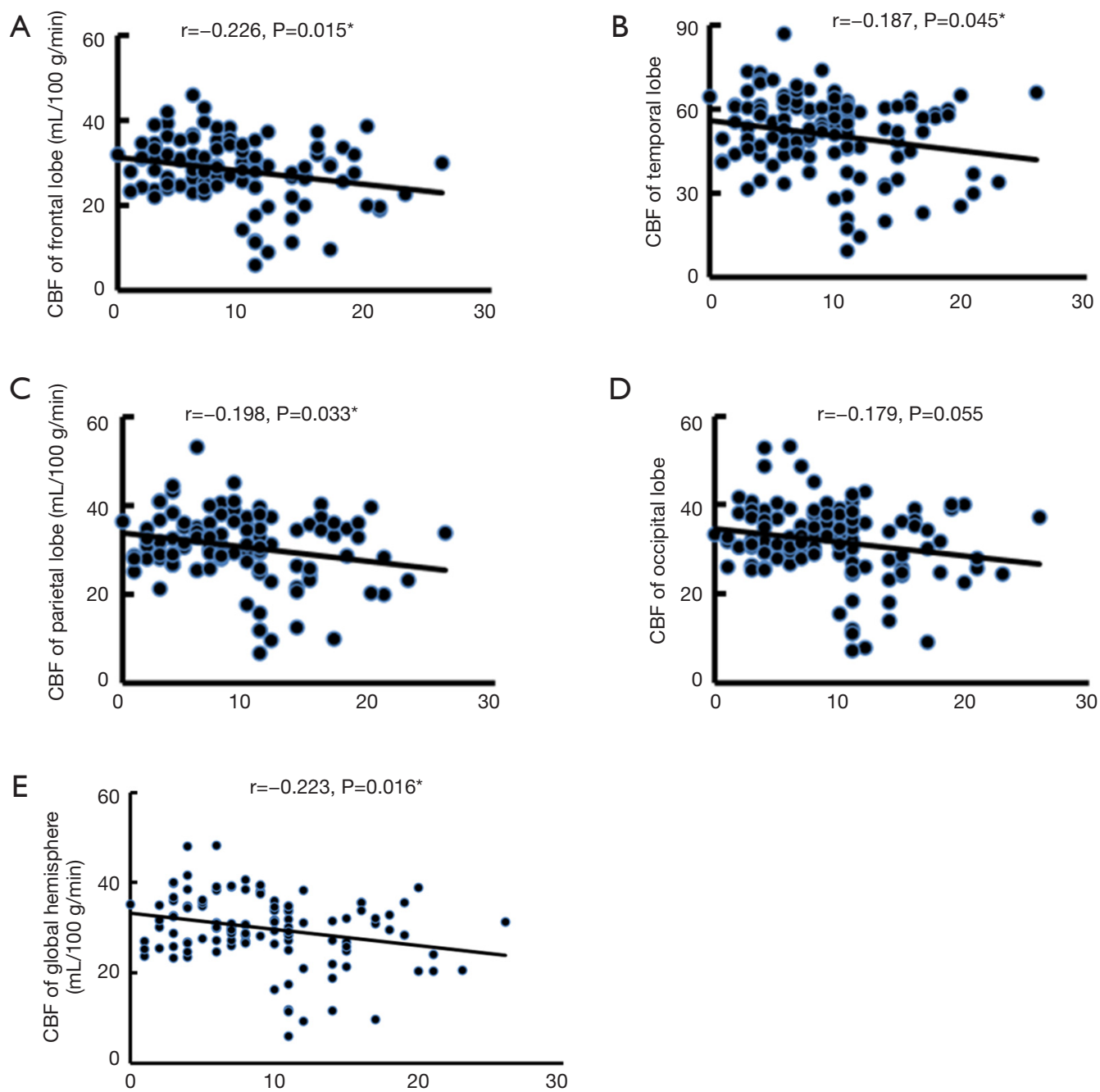

Figure 3 Correlation between CBF and HAMD score. HAMD score negatively correlated with CBFs of the global hemisphere (E), frontal lobe (A), temporal lobe (B), parietal lobe (C), and occipital lobe (D). *, $\mathrm{P}<0.05$.

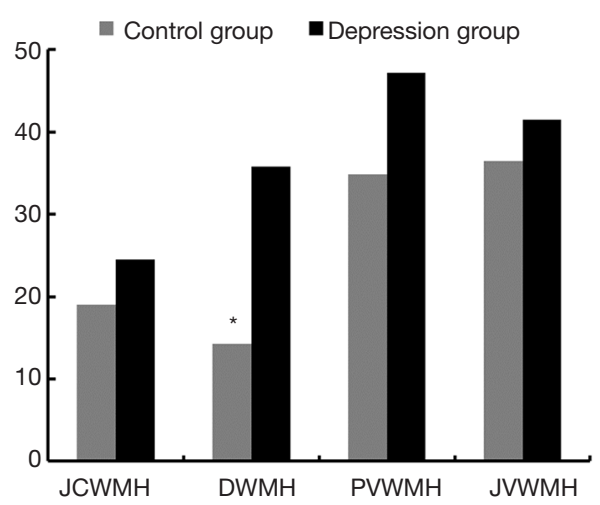

Figure 4 Comparison of the percentage of moderate-to-severe WMH between the control group and LOD group. *, $\mathrm{P}<0.05$. significant stenosis of MCAs in LOD participants compared to the control group, indicating that severe macrovascular pathology was involved. Significant hypoperfusion in the frontal and parietal lobes could be a consequence of MCA stenosis. Consistent with previous studies that showed an association between global or regional cerebral hypoperfusion and vascular depression (23), our findings indicated that reduced $\mathrm{CBF}$ worsens with LOD progression. The combination of a low global or regional perfusion status and moderate-to-severe stenosis of MCAs suggests a diffuse or localized cerebral microand macrovascular pathology of LOD. Regional or global perfusion deficits could disrupt the steady supply of oxygen and nutrients to the brain, adversely affecting gray matter 
function and resulting in the development of WMHs. These pathophysiological changes further distort regional brain structure and possibly disrupt emotional regulation in vascular depression $(12,38)$.

WMH loads as imaged by MR are considered the image phenotype of ischemic damage to cerebral small vessels $(39,40)$ and are interpreted to be small vessel cerebral vascular disease $(41,42)$. WMHs are clinically important because images taken of WMHs can be used to predict poor response to treatment and increased probability of relapse, and WMHs have additionally been suggested as a reliable biological risk marker for late-onset mood disorders (43). WMHs are commonly associated with advanced age and other cerebrovascular risk factors $(31,44)$. WMH loads have been found to be associated with regional cerebral blood volume (45). Compared with the cerebral cortex, DWM is poorly vascularized. It is supplied by long penetrating and perforating arteries and medullary arteries arising from short branches of the MCAs. The arteries supplying cerebral white matter do not usually anastomose, which makes DWM more vulnerable to hypoperfusion from MCAs. A recent study using TCD has suggested severe WMH loads likely arise from small vessels and then extend into larger arteries (23). Our data showed that both large arteries and small vessels were significantly involved in LOD. Damage to large and small vessels may or may not occur simultaneously, depending on the stage and severity of disease. Stenotic intracranial large arteries and small vessels can result in deficient blood supply to DWM, supporting the concept that DWMHs have a primarily vascular etiology (46). The affected microvessels are presumed to induce lesions in DWM and disrupt the bloodbrain barrier (BBB), leading to chronic leakage of plasma into white matter (47).

It is still controversial whether lesion location or the severity of WMHs contribute to either the pathogenesis or aggravation of LOD, or vascular depression. Several studies have suggested that the location of cerebral WMHs plays a prominent role in the pathogenesis of $\operatorname{LOD}(13,40)$, while other studies have shown that WMH severity is a more valuable marker for broader deficits in microvascularity and autoregulation $(48,49)$. Our study compared the importance of both the severity and location of WMHs in LOD pathogenesis using Kim's method. To date, Fazekas' method (50) has been widely used to subdivide WMHs into JVWMH and DWMH. However, this subdivision of WMHs is not able to reflect pathophysiological changes within regions due to the high heterogeneity of $\mathrm{WMH}$ loads. The Fazekas scale (50) divides WMHs into two types: paraventricular and deep lesions. It is simple and practical, but also has some defects, primarily because it is too general to distinguish WMHs present in different parts of the brain. White matter fibers receive varying blood supply and have varying functions depending on where they are located in the brain. Therefore, a more practical method is needed to localize and explore WMHs. The Kim scale (29) is currently the most suitable method for clinical needs. In this scale, WMH is divided into four types depending on the lesion location and blood supply arteries. Kim's method was therefore applied in this study to subdivide WMHs into JVWMH, PVWMH, DWMH, and JCWMH. Our results showed that, among these four subdivisions of WMH, there was a significant difference in high DWMH loads (moderate-to-severe WMHs) between the depression and control groups as determined by FLAIR MRI. This finding confirms that both the severity and location of WMH loads play an important role in LOD development. Consequently, moderate-to-severe WMH loads in DWM could serve as a key imaging marker to indicate broader dysfunction in microvascularity and autoregulation in LOD.

This study provides evidence that both intracranial macrovascularity and microvascularity can be impaired in LOD, and we cautiously conclude that both intracranial large arterial stenosis, particularly in MCA, and moderateto-severe DWM lesion load in the frontal and parietal lobes contribute to the development of LOD. This study may therefore provide reliable radiological hallmarks to define LOD. LOD might be caused by continuous hypoperfusion in vulnerable cerebral regions, such as DWM in the frontal and parietal lobes. This hypoperfusion may disrupt regional or global autoregulation, along with age-affected BBB, which is a dynamic physical, transportive, and enzymatic barrier between the brain parenchyma and circulating blood. Disruptions to the BBB can cause leakages and redistribution of neurotransmitters.

The major limitation of this study is that the severity of intracranial large arterial stenosis, such as MCA stenosis, is heterogeneous, while the sample size of each group was not sufficiently large to draw definitive conclusions. For example, fewer patients with moderate-to-severe stenotic MCAs were enrolled in our study compared to mild stenotic MCAs, yet there was a significant difference in the percentage of moderate-to-severe stenotic MCAs between the two groups. In addition, cerebral hypoperfusion and WMHs are frequently reported in the aging population and patients with cardiovascular diseases $(51,52)$. These 
pathological changes are not unique to depressive disorders in the elderly population. Overall, however, our study provides a deeper understanding of the associations between lesions in dominant brain regions, regional or global hypoperfusion, and their potential clinical consequences.

\section{Conclusions}

This study highlights the role of multimodality imaging in the objective assessment of impaired hemodynamics in LOD. We found that a low global or regional perfusion status, moderate-to-severe stenosis of MCAs, and high WMH loads constitute a set of imaging hallmarks for LOD diagnosis.

\section{Acknowledgments}

Funding: The authors gratefully acknowledge the support of the National Key Research and Development Program of China (2018YFC1314700), the National Science Foundation of China (No. 81671307), and Priority of Shanghai Key Discipline of Medicine (2017ZZ02020).

\section{Footnote}

Conflicts of Interest: All authors have completed the ICMJE uniform disclosure form (available at http://dx.doi. org/10.21037/qims-19-402). The authors have no conflicts of interest to declare.

Ethical Statement: The study was conducted in accordance with the Declaration of Helsinki (as revised in 2013). This study was reviewed and approved by the Institutional Review Board at Tongji University and written informed consent was obtained from all patients.

Open Access Statement: This is an Open Access article distributed in accordance with the Creative Commons Attribution-NonCommercial-NoDerivs 4.0 International License (CC BY-NC-ND 4.0), which permits the noncommercial replication and distribution of the article with the strict proviso that no changes or edits are made and the original work is properly cited (including links to both the formal publication through the relevant DOI and the license). See: https://creativecommons.org/licenses/by-nc-nd/4.0/.

\section{References}

1. Steffens DC. Establishing diagnostic criteria for vascular depression. J Neurol Sci 2004;226:59-62.

2. Román GC. Vascular Depression: An Archetypal Neuropsychiatric Disorder. Biol Psychiatry 2006;60:1306-8.

3. Hategan A, Bourgeois JA, Hirsch CH, Giroux C (Eds). Geriatric Psychiatry. Late-Life Depression. Springer International Publishing, 2018.

4. Bella R, Pennisi G, Cantone M, Palermo F, Pennisi M, Lanza G, Zappia M, Paolucci S. Clinical presentation and outcome of geriatric depression in subcortical ischemic vascular disease. Gerontology 2010;56:298-302.

5. Driscoll HC, Basinski J, Mulsant BH, Butters MA, Dew MA, Houck PR, Mazumdar S, Miller MD, Pollock BG, Stack JA, Schlernitzauer MA, Reynolds CF 3rd. Lateonset major depression: clinical and treatment-response variability. Int J Geriatr Psychiatry 2005;20:661-7.

6. Baldwin RC, O'Brien J. Vascular basis of late-onset depressive disorder. Br J Psychiatry 2002;180:157-60.

7. Salo KI, Scharfen J, Wilden ID, Schubotz RI, Holling H. Confining the Concept of Vascular Depression to LateOnset Depression: A Meta-Analysis of MRI-Defined Hyperintensity Burden in Major Depressive Disorder and Bipolar Disorder. Front Psychol 2019;10:1241.

8. Santos M, Xekardaki A, Kövari E, Gold G, Bouras C, Giannakopoulos P. Microvascular pathology in late-life depression. J Neurol Sci 2012;322:46-9.

9. Yang YH, Chiu CC, Teng HW, Huang CT, Liu CY, Huang LJ. Aspirin and Risk of Dementia in Patients with Late-Onset Depression: A Population-Based Cohort Study. Biomed Res Int 2020;2020:1704879-.

10. Vink D, Aartsen MJ, Schoevers RA. Risk factors for anxiety and depression in the elderly: A review. J Affect Disord 2008;106:29-44.

11. Krishnan KRR. Biological risk factors in late life depression. Biol Psychiatry 2002;52:185-92.

12. Krishnan KRR, Taylor WD, McQuoid DR, MacFall JR, Payne ME, Provenzale JM, Steffens DC. Clinical characteristics of magnetic resonance imaging-defined subcortical ischemic depression. Biol Psychiatry 2004;55:390-7.

13. Taylor WD, MacFall JR, Steffens DC, Payne ME, Provenzale JM, Krishnan KRR. Localization of ageassociated white matter hyperintensities in late-life depression. Prog Neuropsychopharmacol Biol Psychiatry 2003;27:539-44.

14. Figiel GS, Krishnan KRR, Murali Doraiswamy P, Rao VP, Nemeroff CB, Boyko OB. Subcortical hyperintensities on brain magnetic resonance imaging: A comparison between 
late age onset and early onset elderly depressed subjects. Neurobiol Aging 1991;12:245-7.

15. Park JH, Lee SB, Lee JJ, Yoon JC, Han JW, Kim TH, Jeong HG, Newhouse PA, Taylor WD, Kim JH, Woo JI, Kim KW. Epidemiology of MRI-defined vascular depression: A longitudinal, community-based study in Korean elders. J Affect Disord 2015;180:200-6.

16. Li J, Yang Y, Zhu Y, Zhou L, Han Y, Yin T, Cheng Z, Zhang G, Shen Y, Chen J. Towards characterizing the regional cerebral perfusion in evaluating the severity of major depression disorder with SPECT/CT. BMC Psychiatry 2018;18:70.

17. Wang Y, Liu X, Li P, Zhou H, Yang L, Zheng L, Xie P, Li L, Liao DJ, Liu Q, Fang D. Regional Cerebral Blood Flow in Mania: Assessment Using 320-Slice Computed Tomography. Front Psychiatry 2018;9:296.

18. Wang Y, Zhang H, Tang S, Liu X, O'Neil A, Turner A, Chai F, Chen F, Berk M. Assessing regional cerebral blood flow in depression using 320-slice computed tomography. PLoS One 2014;9:e107735.

19. Bella R, Ferri R, Cantone M, Pennisi M, Lanza G, Malaguarnera G, Spampinato C, Giordano D, Raggi A, Pennisi G. Motor cortex excitability in vascular depression. Int J Psychophysiol 2011;82:248-53.

20. Concerto C, Lanza G, Cantone M, Pennisi M, Giordano D, Spampinato C, Ricceri R, Pennisi G, Aguglia E, Bella R. Different patterns of cortical excitability in major depression and vascular depression: a transcranial magnetic stimulation study. BMC Psychiatry 2013;13:300.

21. Pennisi M, Lanza G, Cantone M, Ricceri R, Spampinato C, Pennisi G, Di Lazzaro V, Bella R. Correlation between Motor Cortex Excitability Changes and Cognitive Impairment in Vascular Depression: Pathophysiological Insights from a Longitudinal TMS Study. Neural Plast 2016;2016:8154969.

22. Nomura Y, Faegle R, Hori D, Al-Qamari A, Nemeth AJ, Gottesman R, Yenokyan G, Brown C, Hogue CW. Cerebral Small Vessel, But Not Large Vessel Disease, Is Associated With Impaired Cerebral Autoregulation During Cardiopulmonary Bypass: A Retrospective Cohort Study. Anesth Analg 2018;127:1314-22.

23. Puglisi V, Bramanti A, Lanza G, Cantone M, Vinciguerra L, Pennisi M, Bonanno L, Pennisi G, Bella R. Impaired Cerebral Haemodynamics in Vascular Depression: Insights From Transcranial Doppler Ultrasonography. Front Psychiatry 2018;9:316-.

24. Charlson M, Peterson JC. Medical comorbidity and late life depression: what is known and what are the unmet needs? Biol Psychiatry 2002;52:226-35.

25. Liu M, Nie ZY, Li RR, Zhang W, Wang H, He YS, Zhao LJ, Li YX. Correlation of Brain Perfusion with White Matter Hyperintensity, Brain Atrophy, and Cognition in Patients with Posterior Cerebral Artery Stenosis and Subjective Cognitive Decline. Med Sci Monit 2018;24:5729-38.

26. Li RR, He YS, Liu M, Nie ZY, Huang LH, Lu Z, Jin LJ, Li YX. Analysis of correlation between cerebral perfusion and KIM score of white matter lesions in patients with Alzheimer's disease. Neuropsychiatr Dis Treat 2019;15:2705-14.

27. Wang H, Nie ZY, Liu M, Li RR, Huang LH, Lu Z, Jin LJ, Li YX. Clinical characteristics of perivascular space and brain CT perfusion in stroke-free patients with intracranial and extracranial atherosclerosis of different extents. Ann Transl Med 2020;8:215.

28. Samuels OB, Joseph GJ, Lynn MJ, Smith HA, Chimowitz MI. A standardized method for measuring intracranial arterial stenosis. AJNR Am J Neuroradiol 2000;21:643-6.

29. Kim KW, MacFall JR, Payne ME. Classification of White Matter Lesions on Magnetic Resonance Imaging in Elderly Persons. Biol Psychiatry 2008;64:273-80.

30. Vinciguerra L, Lanza G, Puglisi V, Pennisi M, Cantone M, Bramanti A, Pennisi G, Bella R. Transcranial Doppler ultrasound in vascular cognitive impairment-no dementia. PLoS One 2019;14:e0216162.

31. Naarding P, Tiemeier H, Breteler MMB, Schoevers RA, Jonker C, Koudstaal PJ, Beekman ATF. Clinically defined vascular depression in the general population. Psychol Med 2007;37:383-92.

32. van Straaten ECW, Scheltens P, Barkhof F. MRI and CT in the diagnosis of vascular dementia. J Neurol Sci 2004;226:9-12.

33. Alexopoulos GS. Vascular disease, depression, and dementia. J Am Geriatr Soc 2003;51:1178-80.

34. Schoevers RA, Beekman ATF, Deeg DJH, Geerlings MI, Jonker C, Van Tilburg W. Risk factors for depression in later life; results of a prospective community based study (AMSTEL). J Affect Disord 2000;59:127-37.

35. Scott R, Paulson D. Cerebrovascular burden and depressive symptomatology interrelate over 18 years: support for the vascular depression hypothesis. Int $\mathrm{J}$ Geriatr Psychiatry 2018;33:66-74.

36. Taylor WD, Aizenstein HJ, Alexopoulos GS. The vascular depression hypothesis: mechanisms linking vascular disease with depression. Mol Psychiatry 2013;18:963-74.

37. Su H, Zuo C, Zhang H, Jiao F, Zhang B, Tang W, 
Geng D, Guan Y, Shi S. Regional cerebral metabolism alterations affect resting-state functional connectivity in major depressive disorder. Quant Imaging Med Surg 2018;8:910-24.

38. Pantoni L, Garcia Julio H. Pathogenesis of Leukoaraiosis. Stroke 1997;28:652-9.

39. Toma S, MacIntosh BJ, Swardfager W, Goldstein BI. Cerebral blood flow in bipolar disorder: A systematic review. J Affect Disord 2018;241:505-13.

40. Thomas AJ, O'Brien JT, Davis S, Ballard C, Barber $\mathrm{R}$, Kalaria RN, Perry RH. Ischemic basis for deep white matter hyperintensities in major depression: a neuropathological study. Arch Gen Psychiatry 2002;59:785-92.

41. Pantoni L. Cerebral small vessel disease: from pathogenesis and clinical characteristics to therapeutic challenges. Lancet Neurol 2010;9:689-701.

42. Wardlaw JM, Smith EE, Biessels GJ, Cordonnier C, Fazekas F, Frayne R, Lindley RI, O'Brien JT, Barkhof F, Benavente OR, Black SE, Brayne C, Breteler M, Chabriat H, Decarli C, de Leeuw FE, Doubal F, Duering M, Fox NC, Greenberg S, Hachinski V, Kilimann I, Mok V, Oostenbrugge Rv, Pantoni L, Speck O, Stephan BC, Teipel S, Viswanathan A, Werring D, Chen C, Smith C, van Buchem M, Norrving B, Gorelick PB, Dichgans M; STandards for ReportIng Vascular changes on nEuroimaging (STRIVE v1). Neuroimaging standards for research into small vessel disease and its contribution to ageing and neurodegeneration. Lancet Neurol 2013;12:822-38.

43. Pompili M, Serafini G, Innamorati M, Serra G, Forte A, Lester D, Ducci G, Girardi P, Tatarelli R. White matter hyperintensities, suicide risk and late-onset affective disorders: an overview of the current literature. Clin Ter 2010;161:555-63.

44. Taylor WD, MacFall JR, Payne ME, McQuoid DR,

Cite this article as: Wang J, Li R, Liu M, Nie Z, Jin L, Lu Z, Li Y. Impaired cerebral hemodynamics in late-onset depression: computed tomography angiography, computed tomography perfusion, and magnetic resonance imaging evaluation. Quant Imaging Med Surg 2020;10(9):1763-1774. doi: 10.21037/qims19-402
Steffens DC, Provenzale JM, Krishnan RR. Greater MRI lesion volumes in elderly depressed subjects than in control subjects. Psychiatry Res 2005;139:1-7.

45. Wen W, Sachdev P, Shnier R, Brodaty H. Effect of white matter hyperintensities on cortical cerebral blood volume using perfusion MRI. Neuro Image 2004;21:1350-6.

46. O’Sullivan M, Lythgoe DJ, Pereira AC, Summers PE, Jarosz JM, Williams SCR, Markus HS. Patterns of cerebral blood flow reduction in patients with ischemic leukoaraiosis. Neurology 2002;59:321.

47. Topakian R, Barrick TR, Howe FA, Markus HS. Bloodbrain barrier permeability is increased in normalappearing white matter in patients with lacunar stroke and leucoaraiosis. J Neurol Neurosurg Psychiatry 2010;81:192.

48. DeCarli C, Murphy DG, Tranh M, Grady CL, Haxby JV, Gillette JA, Salerno JA, Gonzales-Aviles A, Horwitz B, Rapoport SI. The effect of white matter hyperintensity volume on brain structure, cognitive performance, and cerebral metabolism of glucose in 51 healthy adults. Neurology 1995;45:2077-84.

49. Markus HS, Lythgoe DJ, Ostegaard L, O'Sullivan M, Williams SC. Reduced cerebral blood flow in white matter in ischaemic leukoaraiosis demonstrated using quantitative exogenous contrast based perfusion MRI. J Neurol Neurosurg Psychiatry 2000;69:48-53.

50. Fazekas F, Chawluk JB, Alavi A, Hurtig HI, Zimmerman RA. MR signal abnormalities at $1.5 \mathrm{~T}$ in Alzheimer's dementia and normal aging. AJR Am J Roentgenol 1987;149:351-6.

51. Camus V, Kraehenbühl H, Preisig M, Büla CJ, Waeber G. Geriatric depression and vascular diseases: what are the links? J Affect Disord 2004;81:1-16.

52. Herrmann LL, Le Masurier M, Ebmeier KP. White matter hyperintensities in late life depression: a systematic review. J Neurol Neurosurg Psychiatry 2008;79:619-24. 